Bio-functional subwavelength optical waveguides for biodetection

Donald J. Sirbuly, Nicholas Fischer, Shih-Chieh Huang, Alexander Artyukhin, Jeff Tok, Olgica Bakajin, Aleksandr Noy

July 16, 2007

ACS Nano 
This document was prepared as an account of work sponsored by an agency of the United States Government. Neither the United States Government nor the University of California nor any of their employees, makes any warranty, express or implied, or assumes any legal liability or responsibility for the accuracy, completeness, or usefulness of any information, apparatus, product, or process disclosed, or represents that its use would not infringe privately owned rights. Reference herein to any specific commercial product, process, or service by trade name, trademark, manufacturer, or otherwise, does not necessarily constitute or imply its endorsement, recommendation, or favoring by the United States Government or the University of California. The views and opinions of authors expressed herein do not necessarily state or reflect those of the United States Government or the University of California, and shall not be used for advertising or product endorsement purposes. 
July 16, 2007

Professor A. Paul Alivisatos

Editor, Nano Letters

University of California

B62 Hildebrand Hall

Berkeley, California 94720-1460

Dear Prof. Alivisatos,

Enclosed please find our manuscript entitled "Biofunctional Subwavelength Optical

Waveguides for Biodetection" that we are submitting for publication in Nano Letters.

In this paper, we demonstrate a biofunctional subwavelength photonic platform capable of real-time biomolecule detection. Our scaffold consists of a subwavelength semiconductor waveguide coated with functional lipid membranes that serves as a matrix for the biomolecule receptor species. This bio-nano platform offers several unique capabilities:

(1) This optical scaffold enables biomolecules to be anchored within the evanescent field for all-optical biomolecular sensing.

(2) The propagating evanescent field of the subwavelength optical waveguides allows highly-efficient real-time optical monitoring (i.e., spectroscopy) of the biomembranes.

(3) The waveguides can be integrated into polymeric flow cells for rapid membrane fusion and removal; allowing full reusability of the optical surface of the waveguides.

Our manuscript reports a novel subwavelength photonic platform that provides a seamless and natural interface between solid inorganic optical materials and soft, biological membranes. Due to their compact probe volumes (sub-femtoliter), reusability, and ability to use various optical sensing modalities, these biofunctional waveguides provide an ideal portable photonic architecture for biomolecular screening and sensing, both in situ and exsitu.

We believe that the results presented in this work would be interesting to the nanoscience research community and to the researchers in the wider biosensing and biomembrane communities; as these highly efficient portable detection platforms could be used for medical diagnostics, environmental monitoring and pathogen detection applications. 
We are attaching a list of experts who can evaluate our manuscript at the end of this letter. If the manuscript is accepted for publication we ask to publish the figures in color, as it would enhance the clarity of the presentation significantly. Thank you for your time in appraising our manuscript for publication in Nano Letters.

Sincerely,

Donald J. Sirbuly and Aleksandr Noy

Donald J. Sirbuly, Ph.D.

Chemistry, Materials, and Life Sciences Directorate

Lawrence Livermore National Laboratory, L-234

7000 East Ave.

Livermore, CA 94550

Fax: (925) 422-2041

Tel: (925) 424-6474

E-mail: sirbuly2@llnl.gov

Aleksandr Noy, Ph.D.

Chemistry, Materials, and Life Sciences Directorate

Lawrence Livermore National Laboratory, L-234

7000 East Ave.

Livermore, CA 94550

Fax: (925) 424-6203

Tel: (925) 424-6474

E-mail: noy1@llnl.gov

\section{Suggested referees:}

Prof. George Whitesides

Department of Chemistry and Chemical Biology

Harvard University

Cambridge, MA

Tel: (617) 495-9430

E-mail: gwhitesides@gmwgroup.harvard.edu

Prof. Charles M. Lieber

Department of Chemistry and Chemical Biology

Harvard University

Cambridge, MA 02138

Tel: (617) 496-4237

Email: cml@cmliris.harvard.edu 
Prof. Harry A. Atwater, Jr.

246 Watson

MC 128-95

Californian Institute of Technology

Pasadena, CA

Tel: (626) 395-2197

E-mail: haa@daedalus.caltech.edu

Prof. Mostafa A. El-Sayed

Department of Chemistry \& Biochemistry

Georgia Institute of Technology

901 Atlantic Drive

Atlanta, GA 30332-0400

Tel: (404) 894-0292

E-mail: mostafa.el-sayed@chemistry.gatech.edu

Prof. Uri Banin

Institute of Chemistry and The Center for Nanoscience and Nanotechnology

The Hebrew University of Jerusalem

Jerusalem, 91904 Israel

Tel: +972-2-6584515

E-mail: banin@chem.ch.huji.ac.il

Prof. Robert M. Dickson

School of Chemistry and Biochemistry

770 State Street - Boggs Building

Georgia Institute of Technology

Atlanta, GA 30332

Tel: (404) 894-4007

E-mail: robert.dickson@chemistry.gatech.edu

Prof. Younan Xia

Department of Chemistry

University of Washington

Seattle, WA 98195-1700

Tel: (206) 543-1767

E-mail: xia@chem.washington.edu

Associate Prof. Peidong Yang

Department of Chemistry

University of California

Berkeley, CA 94720-1460

Tel: (510) 643-1545

E-mail: p_yang@berkeley.edu 


\title{
Biofunctional Subwavelength Optical Waveguides for Biodetection
}

\author{
Donald J. Sirbuly*, Nicholas O. Fischer, Shih-Chieh J. Huang, \\ Alexander B. Artyukhin, Jeffrey B.-H. Tok, Olgica Bakajin, and \\ Aleksandr Noy* \\ Chemistry, Materials, and Life Sciences Directorate \\ Lawrence Livermore National Laboratory, 7000 East Ave., Livermore, CA 94550 \\ *Address correspondence to D.J.S. and A.N. ( sirbuly2@llnl.gov, noy1@llnl.gov)

\begin{abstract}
We report a versatile biofunctional subwavelength photonic device platform for real-time detection of biological molecules. Our devices contain lipid bilayer membranes fused onto metal oxide nanowire waveguides stretched across polymeric flow channels. The lipid bilayers incorporating target receptors are submersed in the propagating evanescent field of the optical cavity. We show that the lipid bilayers in our devices are continuous, have very high mobile fraction, and are resistant to fouling. We also demonstrate that our platform allows rapid membrane exchange. Finally we use this device for detection of specific DNA sequences in solution by anchoring complementary DNA target strands in the lipid bilayer. This evanescent wave sensing architecture holds great potential for portable, all-optical detection systems.
\end{abstract}

Designing advanced optical components for biological diagnostics will require interfacing the cellular environments with photonic materials. Supported phospholipid bilayer membranes represent a versatile, robust, and increasingly common approach for achieving biological compatibility for materials surfaces. ${ }^{1-3}$ Because of the central role the lipid bilayers play in a variety of important cellular processes, ${ }^{4}$ such as molecular transport, signaling and recognition they provide almost limitless possibilities for integrating living biosystems with manmade materials and devices. For all-optical, chip-based biochemical sensing and analysis, lipid membranes represent a viable and highly-versatile means for 
functionalizing an active sensing element with target receptors. Non-fouling properties of the lipid membrane also enable efficient rejection of non-specific interactions. ${ }^{1}$

The ease of fabricating a continuous lipid membrane by vesicle fusion is well documented.3, 5, 6 This process allows efficient formation of a bilayer on a wide variety of support materials, ${ }^{3}$ geometries, ${ }^{7}$ and curvatures $^{8}$ in a matter of seconds to minutes. It is also extremely flexible in producing membranes with various chemical compositions. For example, membranes containing charged phospholipids, glycolipids, and membrane-linked or membrane-embedded proteins can be created under virtually the same synthetic conditions. ${ }^{9}, 10$ These characteristics make lipid membranes an ideal environment for integration with subwavelength optical components.

The next generation of ultra-sensitive optical biosensor strategies has to rely on materials and structures that can efficiently deliver photons to a small probing area. Recently, Craighead and co-workers demonstrated single molecule spectroscopy using lipid membrane coated zero-mode waveguides. ${ }^{11}$ Although volume confinements can reach the zeptoliter $\left(10^{-21} \mathrm{~L}\right)$ range, enabling single molecule analysis at biological concentrations, the non-propagating optical field makes simultaneous readouts and multiplexing difficult. High aspect ratio $\left(>10^{3}\right)$ subwavelength semiconductor nanowires ${ }^{12}$ represent an alternative class of materials that can support a propagating optical wave for spectroscopic analysis, and can sustain single inputs for manifold analyzers. Tin dioxide $\left(\mathrm{SnO}_{2}\right)$ nanowires are one example of a one-dimensional semiconductor waveguide that has subwavelength rectangular cross-sections ranging from 100 to $400 \mathrm{~nm}$. These dimensions allow up to $30 \%$ of the confined optical energy to travel outside the waveguide in the surrounding cladding which offers a unique platform for designing miniaturized optical sensors. In fact, the optical power residing near the surface of these subwavelength optical cavities, also known 
as the evanescent field, is sufficient enough to carry out complementary spectroscopic techniques, such as fluorescence, absorption, and surface enhanced Raman spectroscopy (SERS), on femtoliter probe volumes passing through the evanescent field. ${ }^{13}$

Here we describe a versatile generalized strategy for biocompatible functionalization of free-standing subwavelength $\mathrm{SnO}_{2}$ waveguides integrated into a polymeric flow cell by assembling a fluid lipid membrane directly on the waveguide surface. Under slow flow conditions, lipid vesicles fuse on the waveguide surfaces within seconds to form freely mobile bilayers. Rapid cleaning $(<5 s)$ of the waveguides and fast lipid exchange demonstrate the possibility of creating a robust and reusable optical sensing platform based on these nanowire waveguides. Finally, we show that these devices can function as biomolecular sensor by anchoring oligonucleotide strands within the lipid bilayer and observing real-time hybridization of complementary single-stranded DNA (cDNA) species. These results demonstrate the possibility of encoding nanophotonic materials for biochemical detection and lay the groundwork for all-optical in situ biological monitoring.

We chose to use single-crystalline tin dioxide $\left(\mathrm{SnO}_{2}\right)$ nanowire waveguides as the subwavelength optical cavities because of their exceptional combination of optical, mechanical and structural properties that enable simple device integration. $\mathrm{SnO}_{2}$ is a wide band gap semiconductor $\left(E_{g}=3.6 \mathrm{eV}, 344 \mathrm{~nm}\right)$ and a transparent metal oxide with a refractive index ( $n$ ) of 2.1 (for visible wavelengths), making it ideal for optical confinement within the visible and near ultraviolet frequency ranges. ${ }^{14}$ The high index of refraction, compared to silica $(n \sim 1.45)$, allows light to be shuttled around an optical chip surrounded by silica or polymer fluidic channels without significantly increasing propagation losses. In addition, their lengths, dimensions and mechanical flexibility facilitate waveguide transfer to a secondary substrate or device. We use micromanipulation to transport the nanowires 
to an elastomeric flow cell made from poly(dimethylsiloxane) (PDMS) where the waveguides traverse across $50 \mu \mathrm{m}$ wide by $25 \mu \mathrm{m}$ deep channels (see Supporting Information - device fabrication). To provide optimal power in the evanescent field, while maintaining diffraction-limited dimensions, we only used waveguides with diameters of 150 - $200 \mathrm{~nm}$ (see Supporting Information - optical microscopy section). After sealing the device with a fused silica cover slip, the waveguides lie suspended over the microchannel, approximately 5-10 $\mu \mathrm{m}$ from the glass substrate, thereby giving the analyte full access to the surface of the $\mathrm{SnO}_{2}$ cavity (Figure 1a). The sealed devices formed a robust, small and portable package (Figure 1b) that could be interrogated with a variety of optical techniques (Figure 1c).

DOPC lipid vesicles containing $1 \%$ of the fluorescently-labeled lipid marker NBDPE rapidly fused with the waveguide surface (see Figure $3 \mathrm{~b}$ ), both when the lipid solution was dropped onto the waveguide surface, or pulled through the sealed microchannel with the waveguide stretching across it. Optical images of the waveguide surface (Figure 1e or Figure 2a) show that this procedure produces continuous and uniform lipid coatings, while the optical spectrum (Figure S1, Supporting Information) confirms that the fluorescence visible in Figure 2 is coming from the dye-labeled lipid molecules.

A critical feature that defines a functional lipid membrane is the ability of individual lipid molecules to diffuse rapidly along the support surface within the bilayer plane. To test the continuity and determine the diffusivity of our membranes on the $\mathrm{SnO}_{2}$ surface we carried out fluorescence recovery after photobleaching (FRAP) experiments. ${ }^{15} \quad \mathrm{SnO}_{2}$ nanowire waveguides showed an average recovery of ca. $75 \%$ after photobleaching even after multiple bleaching cycles (Figure 2b). The images in Figure 2a illustrate that only minimal fluorescence signal was lost from the bleaching area $\left(\sim 0.2 \mu \mathrm{m}^{2}\right)$ after 5 cycles. We used a 
previously described one-dimensional diffusion model ${ }^{8}$ to determine the lipid mobility from the recovery kinetics. The model assumes that the focused laser spot uniformly bleaches all lipid molecules around the waveguide and that the recovery occurs only from directions parallel to the wire axis. As our waveguides are substantially longer than the bleaching spot size and characteristic diffusion length during bleaching, we can treat the unbleached areas of the cavities as large inexhaustible lipid reservoirs. For the native $\mathrm{SnO}_{2}$ surface supported DOPC membranes we obtain a diffusion constant of $2-5 \cdot 10^{8} \mathrm{~cm}^{2} / \mathrm{s}$, which is nearly identical to fluid bilayers absorbed to glass substrates. ${ }^{16}$ This comparison demonstrates that lipid bilayers form very high quality coatings on the bare $\mathrm{SnO}_{2}$ nanowire surfaces.

Exciting the lipid molecules with the evanescent field rather than directly with the laser spot suppresses the background fluorescence (Figure 3a) and eliminates the need for using a clean, uncoated cover glass and scanning microscope. The kinetic curve of the lipid bilayer fusion (Figure 3b) shows that within 5 seconds (at a flow rate of $\sim 10 \mathrm{~mL} / \mathrm{h}$ ) the fluorescence intensity reaches a maximum and stabilizes as pure buffer removes excess lipid vesicles from the waveguide. The optical images of the fusion process show a slight darkening near the center of the waveguide. Since the optical cavities reside only a few microns below the fused silica cover glass they can bend upwards and stick to the glass. This causes a weaker signal due to fewer lipid molecules adhering between the waveguide and cavity. The $\mathrm{SnO}_{2}$ waveguides are extremely flexible (note the slight curvature in the image plane) and a strong liquid pulse can release the waveguide from the glass to allow complete bilayer coverage (see Figure 3c).

The use of amphiphilic solvents such as detergents or alcohols is a reliable means of removing the lipid bilayer from the waveguide and restoring the initial optical surface. We were able to remove the lipid bilayers from the waveguide by passing a short pulse of pure 
ethanol through the fluid cell (Figure 3c). The alcohol quickly desorbs the lipid membrane in $<1$ second and returns the fluorescence intensity to background levels. Using this protocol, membranes can be rapidly exchanged with a solution pulse train consisting of lipid vesicles, pure buffer, and alcohol. This membrane exchange procedure is robust and can be repeated multiple times (Figure 3d) without any degradation of the waveguide device. Significantly, the composition of the membranes can be changed during these exchanges, providing an additional flexibility for the waveguide chemical modification. Strong acids (e.g. aqua regia solution) can also be used to strip off any contaminants residing on the optical surface after repetitive experimental runs. We have repeatedly cleaned the $\mathrm{SnO}_{2}$ surface with a solution containing 1 part concentrated hydrochloric and nitric acid (ratio of 1:3) to 3 parts water without noticing any decrease in the optical performance of the waveguide.

Lipid membranes are excellent scaffolds for embedding or tethering biological molecules using anchors such as membrane proteins or modified lipids. We used this approach to anchor a short DNA oligonucleotide (21 nucleotide recognition sequence, $\mathrm{T}_{5}$ spacer) modified with a $3^{\prime}$ cholesterol tail to the bilayer. ${ }^{17}$ It is important to note that we did not observe any fragmentation of the DNA strands during vesicle preparation (see Figure S2, Supporting Information,); therefore we assume that after the insertion of the cholesterol tail into the hydrophobic core of the lipid bilayer, the DNA protrudes into the hydrophilic vesicle core or into the surrounding aqueous medium outside the vesicle (Figure 4a). Fluorescence imaging using a nucleic acid stain YOYO-1 showed that after the vesicle fusion on the waveguide the ssDNA molecules remains anchored to the membrane and form a continuous, mobile bilayer coating on the waveguide (Figure $4 \mathrm{~b}$ ). We have also successfully inserted probe ssDNA into a pure DOPC membrane (no DNA) on the waveguide by flowing cholesterol tagged DNA into the channel after membrane fusion (data not shown). It is clear 
from the confocal fluorescent images in Figure 4c that the recovery is much slower than for the dye-tagged lipids described above and shows a measurable decrease in fluorescence intensity over the bleached section after $\sim 5$ minutes (Figure 4c-inset image 3 ). The FRAP data confirm a slower diffusion constant of $0.1-0.2 \cdot 10^{8} \mathrm{~cm}^{2} / \mathrm{s}$, which is an order of magnitude slower than the diffusion constant measured for the NBDPE-doped DOPC membranes. A slower diffusion coefficient measured for the cholesterol-anchored DNA molecules is expected, since a large charged molecule will move slower through the bilayer due to electrostatic interactions between the charged sugar back-bone of the DNA and the polar head groups of the lipid molecules.-

To demonstrate biomolecular sensing capabilities of our device platform, we monitored real-time hybridization of two complementary 21 base-pair ssDNA sequences. One of the sequences was anchored to the surface of the waveguide-supported bilayer (probe ssDNA) while free target DNA (cDNA) was pulled through the sealed microchannel. DNA used in these experiments was labeled with a PicoGreen dye, which is an excellent dye for differentiating between single- and double-stranded DNA (dsDNA) due to its large fluorescence enhancement upon binding to dsDNA. In free solution (no lipids present), the dye shows an $\sim 30 \mathrm{x}$ and $\sim 100 \mathrm{x}$ enhancement in fluorescence over the free dye when binding to ssDNA and dsDNA, respectively (see Figure S3a, Supporting Information). Similar enhancements in the fluorescence were observed from the oligonucleotide-doped lipid vesicles, displaying an $\sim 3 x$ increase in intensity when the cDNA is injected into the solution (Figure 5b). The hybridization on the lipid bilayer surface is very efficient: over $85 \%$ of the annealing in free solution occurs within the first minute and does not require additional dye to label the hybridized DNA pairs. This efficiency allows us to perform the sensing experiments by tagging only the DNA probe sequence anchored to the lipid bilayer. 
Using the evanescent field to detect hybridization allows us to monitor only those photons that are emitted from the dye molecules residing near the surface of the waveguide (Figure 5a). After the probe DNA-doped bilayer forms on the waveguide, the fluorescence signal reaches a steady state (Figure 5c, timecourses). However, once the front of the solution pulse containing target DNA encounters the waveguide, the intensity increases approximately 1.8 - 2.5 times above the bilayer level, indicating that DNA hybridization has occurred. In contrast, no enhancement occurred in solution when mismatched target DNA was added to the probe DNA (see Figure S3b, Supporting Information). There is a slight reduction in the intensity level after hybridization which could indicate a removal of residual dsDNA molecules (i.e., a small fraction of dsDNA that is not inserted into the bilayer). Additional experiments are being carried out to better understand the cause of this intensity drop.

In general, the intensity enhancements observed for DNA hybridization on the lipidcoated waveguides were ca. $25 \%$ smaller than the intensity enhancement in solution hybridization experiments. One explanation is that the amount of available dye is reduced in the waveguide experiment. Hybridization of the DNA in free solution occurs in the presence of excess PicoGreen, whereas in the waveguide experiments the only accessible PicoGreen dye molecules for the target cDNA are those complexed with the probe ssDNA or the small fraction of dye molecules that remains non-specifically bound to the bilayer surface. Since the hybridization event can conceivably displace a portion of the bound PicoGreen, the overall amount of dye available before and after hybridization is reduced, resulting in a lower intensity enhancement. It is also possible that there is labeled probe DNA sandwiched between the waveguide and inner leaflet of the bilayer that is not accessible to the target DNA. In this configuration the overall enhancement will be lower as only the fraction of the DNA molecules properly positioned at the solvent-exposed face of 
the bilayer are contributing to the fluorescent signal. We note that we have performed extensive tests to verify that hybridization was occurring with bilayer supported probe strands, rather than non-specific bound dsDNA interacting with an uncoated waveguide via electrostatic interactions. Flowing PicoGreen labeled dsDNA over a lipid-free waveguide produced only weak (intensities comparable to the ssDNA doped bilayer) fluorescent signals (data not shown).

In conclusion, we have developed a general protocol for interfacing subwavelength optical waveguides with functional biological membranes. Vesicle fusion produces continuous and mobile lipid membranes situated directly within the propagating evanescent field of a metal oxide nanowire optical cavity. Membranes assembled on the nanowires integrated into polymeric flow cells could be rapidly exchanged by alternating short pulses of organic solvent and vesicle solution. This protocol allows sub-10-second chemical compositional changes to the optical surface. Finally, we showed that the waveguides incorporating ssDNA molecules anchored to the lipid bilayer surface can be used for DNA detection. In these experiments hybridization of the complementary DNA strand to the probe DNA on the waveguide in the presence of a DNA-sensitive dye resulted in the enhancement of the fluorescence signal.

Our results institute several important directions for real-time chip-based optical detection of biological molecules. Such photonic scaffolds are a crucial component for the development of miniaturized multiplexed optical sensors which require individual transducers to be encoded with distinct chemical specificity. The sub-femtoliter probe volumes, reusability and the ability to use various optical sensing modalities, make our biofunctional waveguides an ideal portable photonic platform for biomolecular screening, sensing, and in situ medical diagnostics. 
Acknowledgement. D.J.S. acknowledges the Harold C. Graboske Jr. Fellowship from LLNL. N.O.F. acknowledges the CMLS Directorate Fellowship from LLNL. S.C.J.H. and A.B.A. acknowledge support from the SEGRF fellowship at LLNL. A.N. acknowledges LLNL LDRD program funding. We would like to thank Yinmin Wang and Jim Ferreira for their help in capturing SEM images. This work was performed under the auspices of the U.S. Department of Energy by the University of California, Lawrence Livermore National Laboratory, under Contract No. W-7405-Eng-48.

Supporting Information Available: Materials and Methods and Supplemental Figures S1S3. This materials is available free of charge via the Internet at http://pubs.acs.org. 


\section{References.}

1. Katsaras, J.; Gutberlet, T., Lipid Bilayers: Structure and Interactions. SpringerVerlag: 2001; p 312.

2. Tien, H. T.; Ottova-Leitmannova, A. L., Planar Lipid Bilayers (BLMs) and Their Applications. Elseiver Science: Amsterdam, 2003; p 1043.

3. Sackmann, E. Science 1996, 271, 43-48.

4. Yeagle, P. L., The Structure of Biological Membranes. CRC Press: 2004.

5. Tamm, L. K.; McConnell, H. M. Biophys. J. 1985, 47, 105-113.

6. Evans, E. A. Biophys. J. 1980, 31, 425-431.

7. Groves, J. T.; Ulman, N.; Boxer, S. G. Science 1997, 275, 651-653.

8. Artyukhin, A. B.; Shestakov, A. I.; Harper, J.; Bakajin, O.; Stroeve, P.; Noy, A. J. Am. Chem. Soc. 2005, 127, 7538-7542.

9. Groves, J. T. Curr. Opin. Drug Discovery Dev. 2002, 5, 606-612.

10. Groves, J. T.; Boxer, S. G. Acc. Chem. Res 2002, 35, 149-157.

11. Samiee, K. T.; Moran-Mirabal, J. M.; Cheung, Y. K.; Craighead, H. G. Biophys. J. 2006, 90, 3288-3299.

12. Sirbuly, D. J.; Law, M.; Yan, H. Q.; Yang, P. D. J. Phys. Chem. B 2005, 109, 1519015213.

13. Sirbuly, D. J.; Tao, A.; Law, M.; Fan, R.; Yang, P. D. Adv. Mater. 2007, 19, 61-66.

14. Sirbuly, D. J.; Law, M.; Johnson, J. C.; Goldberger, J.; Saykally, R. J.; Yang, P. D. Science 2004, 305, 1269-1273.

15. Axelrod, D.; Koppel, D. E.; Schlessinger, J.; Elson, E.; Webb, W. W. Biophys. J. 1976, $16,1055-1069$.

16. Tocanne, J. F.; Dupoucezanne, L.; Lopez, A. Prog. Lipid Res. 1994, 33, 203-237.

17. Benkoski, J. J.; Hook, F. J. Phys. Chem. B 2005, 109, 9773-9779. 
A

Figure 1

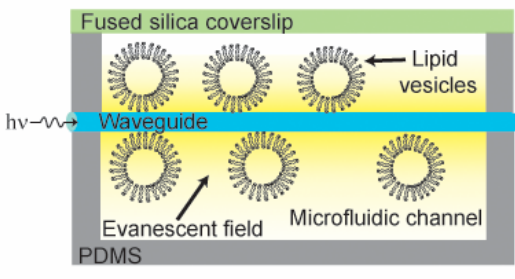

B

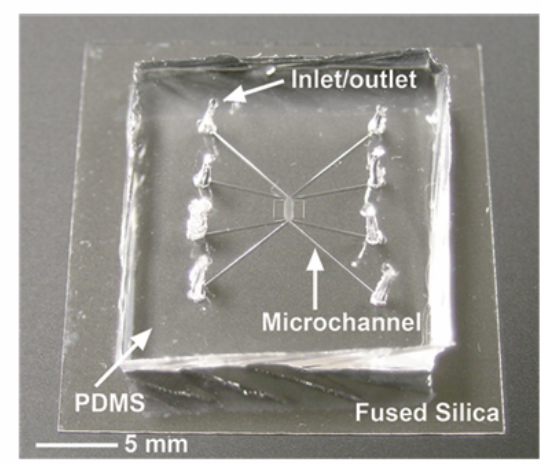

D

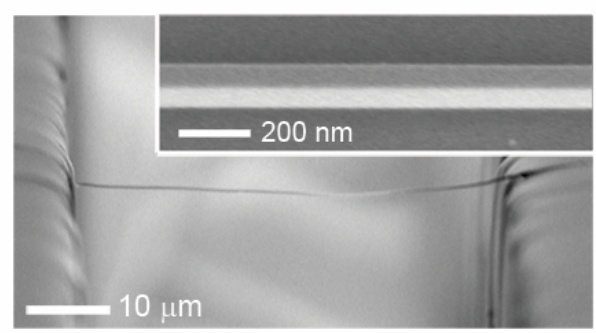

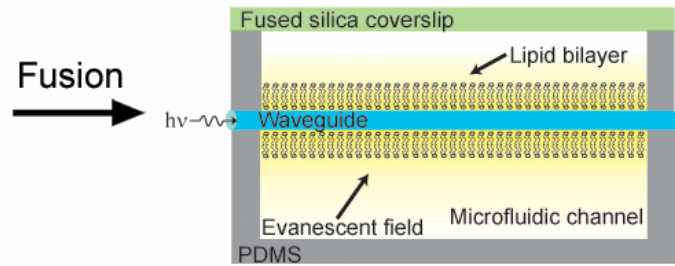

C

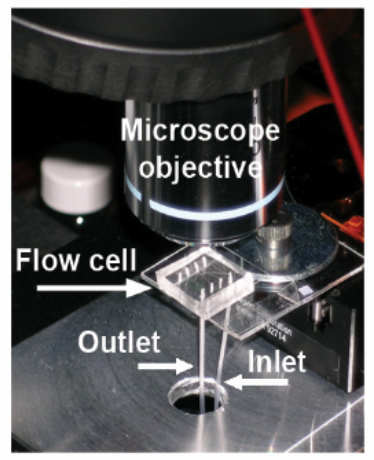

E

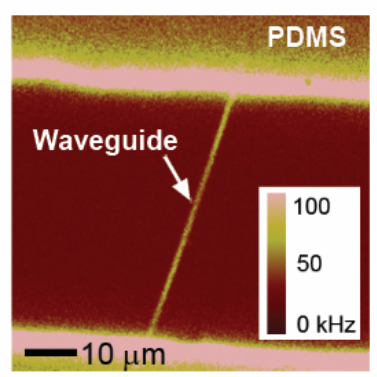

Figure 1. Schematic and layout of the microfluidic flow cell for bilayer formation. (a) Cartoon showing lipid vesicles adsorbing within the evanescent field of a subwavelength waveguide. After fusion a continuous, mobile lipid membrane coats the optical surface. Not shown is the bilayer that forms on the fused silica cover slip and PDMS channel. (b) Digital picture of a PDMS flow cell highlighting the microchannels, glass cover slip, and inlet/outlet holes. (c) Upright microscope set-up for the flow cell. The elastomeric cell sits with its fused silica cover glass facing the microscope objective. (d) Scanning electron micrograph of a waveguide suspended over a microchannel. Inset: Zoom-in SEM image of the surface of a $\mathrm{SnO}_{2}$ waveguide showing the rectangular geometry of the cavity. (e) Confocal optical image ( $80 \times 80 \mu \mathrm{m}$ scan) of a lipid coated waveguide suspended across a PDMS microchannel. 
A
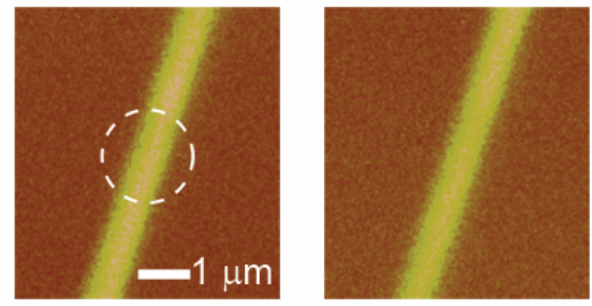

Before bleaching After bleaching

B

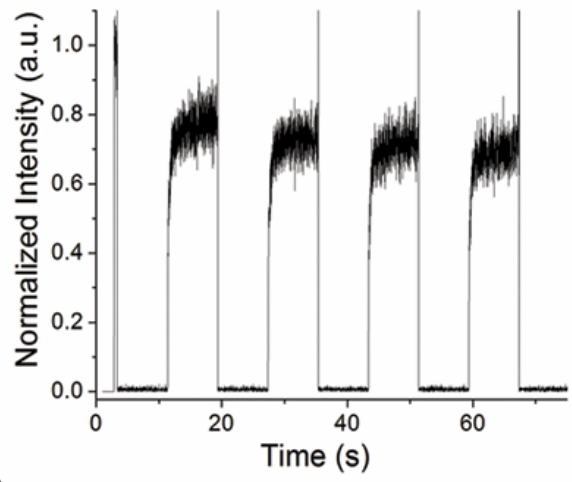

C

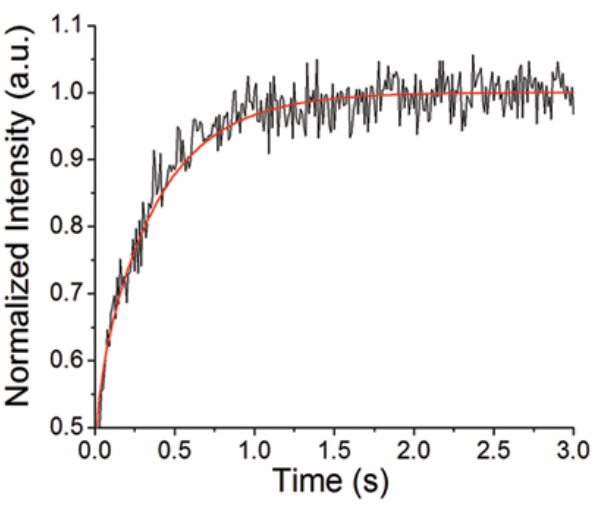

Figure 2. Fluorescence recovery after photobleaching (FRAP) data. (a) Two confocal optical images of a suspended waveguide before and after photobleaching. The lipid bilayer is comprised of DOPC molecules doped with 1\% NBDPDE (fluorescent lipid). The bleached area is denoted by the white dashed circle. (b) FRAP cycling of a bilayer coating a single $\mathrm{SnO}_{2}$ waveguide. The bleach and read laser power densities were held fixed at 12 and $0.12 \mathrm{~kW} / \mathrm{cm}^{2}$, respectively. The sharp spike in counts before the bleach cycle is due to a short delay ( $10 \mathrm{~ms}$ ) between the shutter and EOM.

Even after 5 bleach cycles the bilayers reached an average recovery of $75 \%$ the initial intensity. (c) A single FRAP trace (black curve) with a 1D diffusion model fit (red curve). The diffusion coefficients of the waveguide supported DOPC lipid membranes consistently fell within $2-5 \cdot 10^{8} \mathrm{~cm}^{2} / \mathrm{s}$. 
A
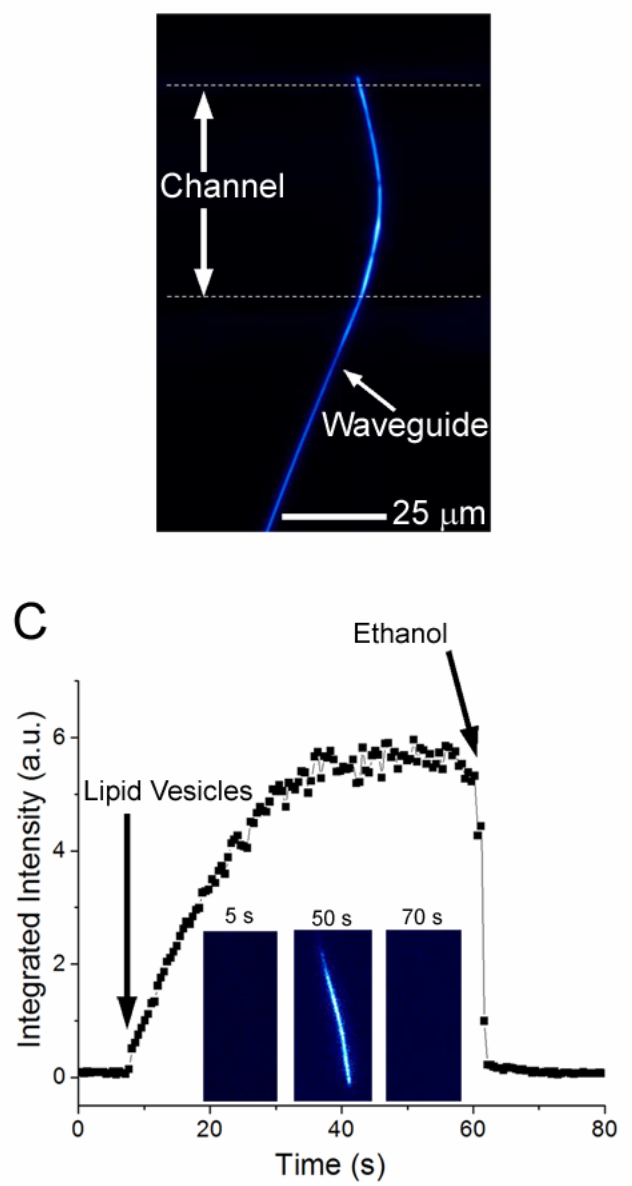

B

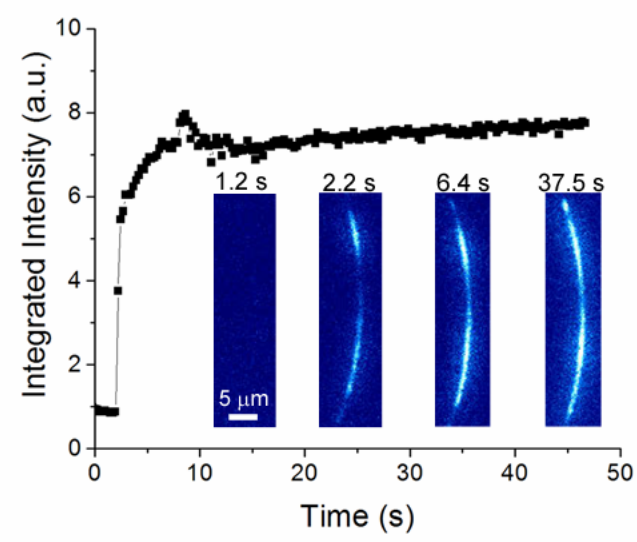

D

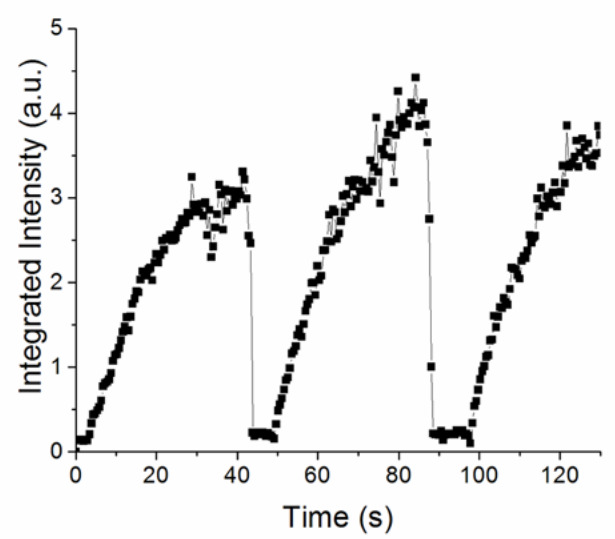

Figure 3. Bilayer fusion and membrane exchange. (a) Dark field optical image of a subwavelength waveguide embedded in a PDMS flow cell. Monochromatic light $(442 \mathrm{~nm})$ is launched into the waveguides at the bottom end terminal (outside field of view). (b) Intensity time course taken from the short waveguide segment traversing the microchannel in (a) as DOPC/NBDPE (99\%/1\%) lipid vesicles (in $10 \mathrm{mM}$ Tris- $\mathrm{HCl}, \mathrm{pH}$ 8) fuse on the $\mathrm{SnO}_{2}$ surface. The optical micrographs show the lipid coverage at discrete times during the fusion process. The evanescent field is exciting the NBDPE lipid molecules within the bilayer. (c) Intensity time course of vesicle fusion ( $<1 \mathrm{~mL} / \mathrm{hr}$ pulling rate) followed by membrane removal with an alcohol (ethanol) liquid pulse. The optical micrographs verify a fused bilayer (time $=50 \mathrm{~s}$ ) and a membrane-free waveguide after cleaning (time $=70 \mathrm{~s}$ ). (d) Intensity time course of multiple lipid-pure solvent-ethanol pulses. Membranes can be rapidly exchanged using sequential liquid pulse trains of lipid and an amphiphilic solvent. 
A
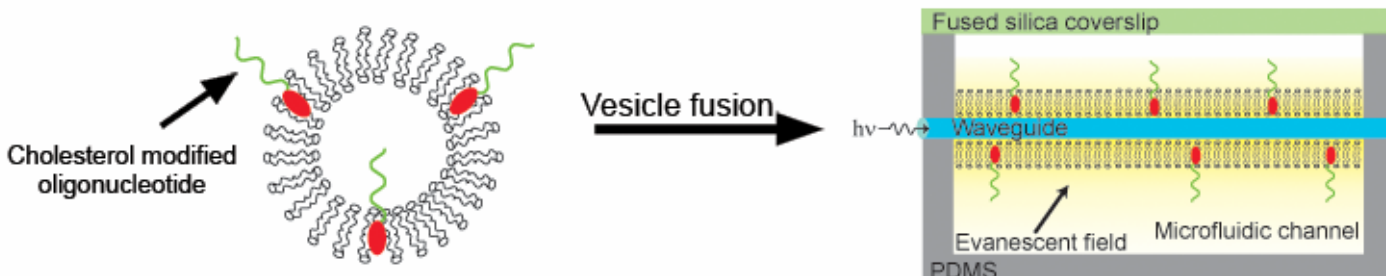

B

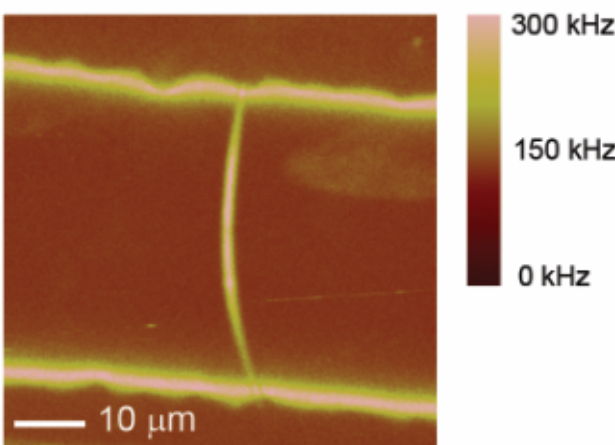

C

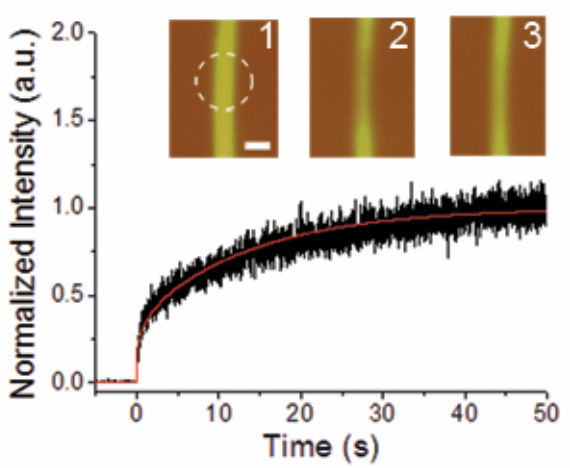

Figure 4. Oligonucleotide doped lipid membranes. (a) Schematic of a DNA doped vesicle and the resulting bilayer. It is possible for some of the strands to point their $5^{\prime}$ ends toward the waveguide instead of into the microchannel (not drawn). (b) Confocal fluorescence image (80 $x 80 \mu \mathrm{m}$ scan) of a YOYO-1 labeled lipid bilayer doped with the probe ssDNA ( $1 \%$ molar ratio doping). The slight bending of the waveguide is a result of the transfer process. The waveguides are extremely flexible (i.e., mechanically) and can sustain high curvature without disrupting bilayer formation. (c) FRAP trace of the DNA doped lipid bilayer showing an order of magnitude slower diffusion $\left(D=0.1-0.2 \cdot 10^{8} \mathrm{~cm}^{2} / \mathrm{s}\right)$ compared to the DOPC/NBDPE membranes. The raw data and 1D diffusion model fit are shown in black and red, respectively. Inset: Sequential confocal images of the same waveguide in (b): (1) before photobleaching,(2) $\sim 2$ minutes post-photobleaching, and (3) $\sim 5$ minutes post-photobleaching. The white dashed circle denotes the approximate position of the laser beam. Scale bar is $500 \mathrm{~nm}$. 
UCRL-JRNL-232761

Figure 5

A

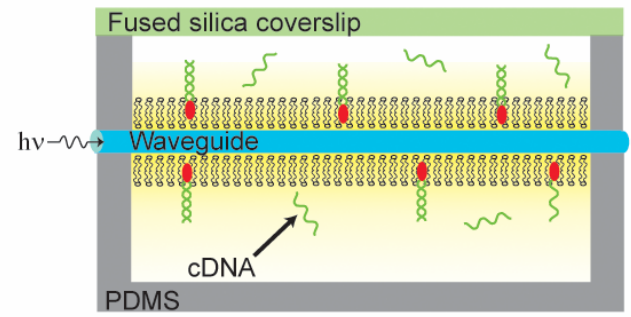

B

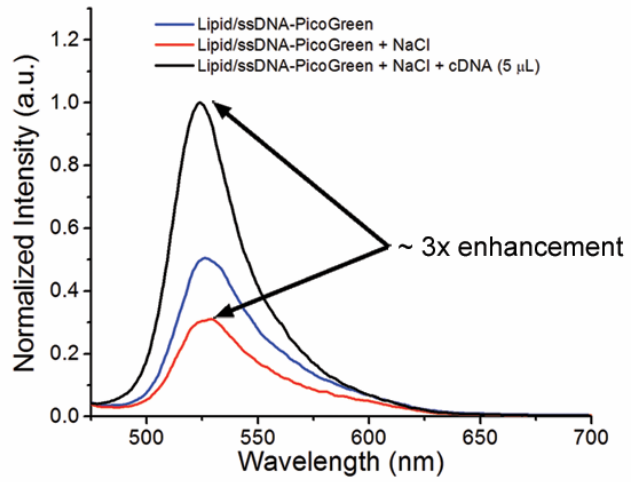

C

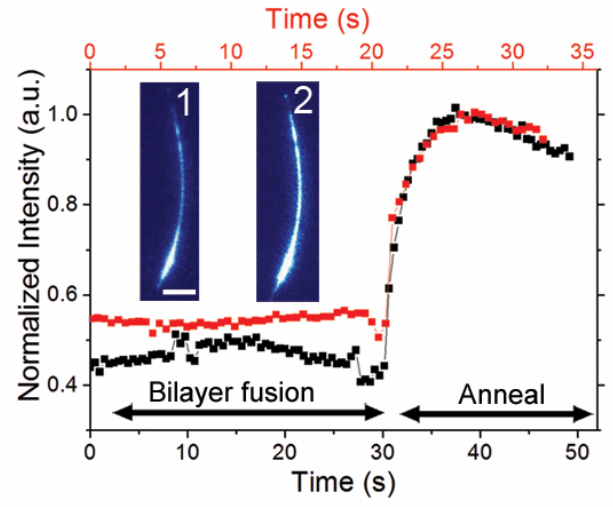

Figure 5. Real-time detection of DNA hybridization. (a) Schematic of annealed DNA anchored by the cholesterol-modified probe strands. The hybridization is monitored by the fluorescence generated by the evanescent field of the optical waveguide. (b) Fluorometry data of DNA doped vesicles in free solution (TBS, pH 8). The data shows an $\sim 40 \%$ decrease in intensity upon salt addition and $\sim 300 \%$ increase after cDNA is injected into the solution. The decrease is well documented for PicoGreen in $\mathrm{NaCl}$ solutions. Spectra are normalized so that the cDNA peak is 1. (c) Two intensity time courses showing hybridization of the target cDNA strands with the probe DNA anchored in the supported lipid membrane. The top (red) and bottom (black) time axes correspond to the red and black traces, respectively. The flow rate was kept below 1 $\mathrm{mL} / \mathrm{hr}$ for 20 seconds prior to annealing to ensure complete bilayer formation and to remove unanchored probe DNA. There is an average of 1.8-2.5x increase in the fluorescence intensity after the target strand encounters the lipid bilayer. Time courses are normalized so the peak integrated intensity is 1 . The optical images are single snap shots taken from a time course; (1) during bilayer formation and (2) after annealing. Scale bar is $10 \mu \mathrm{m}$. 


\section{TOC Graphic}
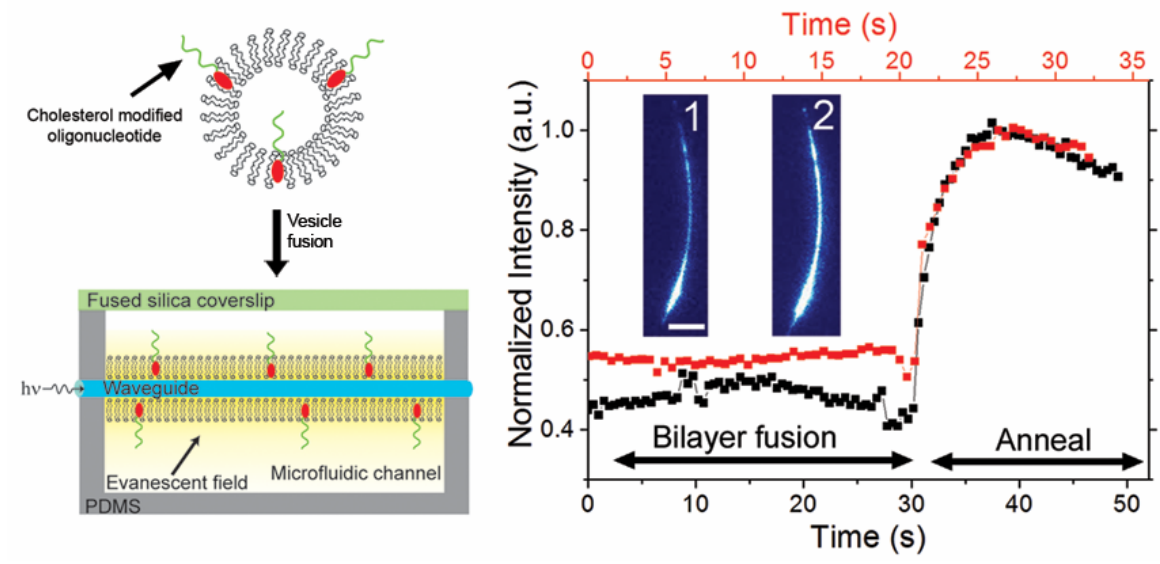


\title{
Biofunctional Subwavelength Optical Waveguides for Biodetection
}

\author{
Donald J. Sirbuly*, Nicholas O. Fischer, Shih-Chieh J. Huang, \\ Alexander B. Artyukhin, Jeffrey B.-H. Tok, Olgica Bakajin, and \\ Aleksandr Noy*
}

Chemistry, Materials, and Life Sciences Directorate

Lawrence Livermore National Laboratory, 7000 East Ave., Livermore, CA 94550

*Address correspondence to D.J.S. and A.N. ( sirbuly2@llnl.gov, noy1@llnl.gov)

\section{Supporting Information}

\section{Materials and Methods}

$\mathrm{SnO}_{2}$ waveguide synthesis. Tin dioxide $\left(\mathrm{SnO}_{2}\right)$ optical waveguides were synthesized by thermally vaporizing tin monoxide powders with trace amounts of oxygen present; similar to previously reported accounts. ${ }^{1,2}$ Briefly, tin monoxide powder (99.9\% Alfa Aesar) was placed in an alumina boat and heated in an alumina tube furnace $\left(\sim 1085{ }^{\circ} \mathrm{C}\right)$ evacuated to 300 mTorr under a $25 \mathrm{sccm}$ flow of argon. After an $\sim 1$ hour growth period, the waveguides were dry transferred from the alumina boat surface to a clean silica surface.

Optical Microscopy. Optical characterization of the sealed and unsealed microfluidic devices was performed with an upright dark-field microscope (see Figure 1c) and inverted laser scanning confocal microscope, respectively. Fluorescence signal was captured with the dark-field microscope using a 50x (Nikon, NA 0.55) objective operating in reflection mode. Signal was either imaged with an EMCCD camera (Andor Technology) or routed to a 
fiber optic coupled spectrometer (Roper Scientific). A continuous-wave (cw) heliumcadmium laser (Kimmon Electric) provided linearly polarized ultraviolet $(325 \mathrm{~nm}$ ) or blue (442 $\mathrm{nm}$ ) radiation for the dark-field microscope. The laser beam was focused to a $\sim 50 \mu \mathrm{m}$ diameter spot onto the sample at an approximate angle of $45^{\circ}$ relative to the sample lying horizontal under the microscope objective. Power densities at the sample ranged from 8 $25 \mathrm{~W} / \mathrm{cm}^{2}$ for the $442 \mathrm{~nm}$ line and $230-270 \mathrm{~W} / \mathrm{cm}^{2}$ for the $325 \mathrm{~nm}$ line, but were held fixed during a single experiment.

The $325 \mathrm{~nm}$ laser line was used to determine the waveguiding properties of the $\mathrm{SnO}_{2}$ nanowire by generating its broad $(380-700 \mathrm{~nm})$ featureless defect emission at one end facet and observing the emission color at the opposing end. According to the waveguide parameter expression $\lambda_{c}=d_{c} \pi / 2.405\left(n_{c o}{ }^{2}-n_{c l}{ }^{2}\right)^{1 / 2}$ for a cylindrical optical cavity, ${ }^{3}$ the singlemode cut-off wavelength $\left(\lambda_{c}\right)$ relates directly to the single-mode cut-off diameter $\left(d_{c}\right)$ of the waveguide and can be used to estimate cross-sectional dimensions of the $\mathrm{SnO}_{2}$ cavity. In general, only waveguides with $\lambda_{c} \leq 600 \mathrm{~nm}$ were used in the devices which corresponds to $d_{c}$ $\leq 250 \mathrm{~nm}$; in good agreement with the actually sizes of the nanowires used here.

The $442 \mathrm{~nm}$ line was used for the evanescent sensing experiments by focusing the laser light on the end facet of the waveguides. Fluorescence generated within the evanescent field was collected by the objective centered over the microfluidic channel and directed to the spectrometer or camera.

The inverted laser scanning confocal microscope imaged the unsealed devices using the $488 \mathrm{nn}$ line (linearly polarized) of an $\mathrm{Ar}^{+}$laser (Spectra-Physics) attenuated to $\sim 120$ $\mathrm{W} / \mathrm{cm}^{2}$ at the sample. The excitation beam was focused to ca. $1 \mu \mathrm{m}$ diameter with a $60 \mathrm{x}$ water immersion objective (Nikon, NA 1.20). The sample was raster scanned across the beam with a nanopositioning stage (Physik Instrumente) controlled by commercially 
available scanning electronics (Digital Instruments). After removing the laser light from the fluorescence, the signal was routed to an avalanche photodiode (APD) and processed by a photon counting board integrated with the scanning electronics.

Device fabrication. Polymeric flow cells were cast from poly(dimethylsiloxane) (PDMS) using a silicon master fabricated using standard lithography techniques. There are four channels running parallel to each other with dimensions $1.25 \mathrm{~mm}$ long x $50 \mu \mathrm{m}$ wide $\mathrm{x} 25$ $\mu \mathrm{m}$ deep, spaced $50 \mu \mathrm{m}$ apart. After curing the elastomer stamp a thin $(\sim 5-10 \mu \mathrm{m})$ wet PDMS layer was placed on the structured side of the stamp. This was achieved by spinning (6000 RPM) uncured PDMS on a glass cover slip and touching the structured side of the stamp to the wet layer. Waveguides optically screened according to the procedure described above were then transferred to the stamps using a 3-axis micromanipulator (Marzhauser Wetzlar) equipped with an etched tungsten probe. Orientation of the cavities was controlled so that the waveguides traverse across the channels leaving one of the end facets accessible for coupling laser light. Devices were sealed for the flow experiments by curing the wet layer after a fused silica cover slip was placed on structured side of the stamp. Curing without a cover slip prepared the sample for confocal fluorescence microscopy. Since the lipid membranes fuse over the entire microchannel, including the glass cover slip, confocal imaging could only be performed with an unsealed device. Strong acids (e.g. aqua regia solution) can also be used to strip off any contaminates residing on the optical surface after repetitive experimental runs. We have repeatedly cleaned the $\mathrm{SnO}_{2}$ surface with a solution containing 1 part concentrated hydrochloric and nitric acid (ratio of 1:3) to 3 parts water without noticing any decrease in the optical performance of the waveguide. 
Lipid bilayer preparation. Unilamellar fluorescent lipid vesicles were prepared via tip probe sonication using a mixture of 1,2-Dioleoyl-sn-Glycero-3-Phosphocholine (DOPC) lipid and a dye tagged lipid 1-Oleoyl-2-[6-[(7-nitro-2-1,3-benzoxadiazol-4-yl)amino]hexanoyl]sn-Glycero-3-Phosphoethanolamine (18:1-06:0 NBDPE) purchased from Avanti Polar Lipids. Appropriate volumes of the lipid stock solution (in chloroform) were transferred to a sample vial to evaporate the chloroform; giving a DOPC/NBDPE molar ratio of 99.0:1.0. After water (or Tris-HCl buffer, see below) was added to the dried lipids, a lipid concentration of $0.3 \mathrm{mg} / \mathrm{mL}(0.1 \mathrm{mg} / \mathrm{mL}$ for the sealed devices $)$ was achieved. The mixtures were tip sonicated at 9 watts for $\sim 1$ minute at room temperature prior to use. Membrane formation on the waveguides was initiated on the sealed devices by pulling (flow rates of $1-10 \mathrm{~mL} / \mathrm{h}$ ) the vesicles over the waveguides for $\sim 1$ min (fusion occurred within seconds) and then flushing the channels with pure buffer to remove excess lipid. For the unsealed devices a small droplet $(\sim 5 \mu \mathrm{L})$ of lipid vesicle solution was placed on the channel side of the stamp for $\sim 5-10$ minutes and then washed with copious amounts of water before placing a clean fused silica cover glass over the microchannels.

Fluorescence Recovery After Photobleaching. Fluorescence recovery after photobleaching (FRAP) experiments were achieved by parking the laser scanning confocal microscope over the center portion of a suspended waveguide. The laser beam was attenuated at a rate faster than the recovery kinetics using an optical train consisting of an electro-optic modulator (EOM - Conoptics) and linear polarizer. The laser intensity was modulated between the bleach $\left(12 \mathrm{~kW} / \mathrm{cm}^{2}\right)$ and read $\left(120 \mathrm{~W} / \mathrm{cm}^{2}\right)$ powers using a function generator (coupled to the EOM) outputting a $62.5 \mathrm{mHz}$ square wave (8 s bleach and read cycles) with an amplitude of $2 \mathrm{~V}_{\mathrm{p}-\mathrm{p}}$. The function generator was synced to an electric shutter so that the APD was blocked during the bleach period and open during the read block. FRAP traces were generated by inputting the raw counts from the APD into a 
counting board (National Instruments). Fits to the recovery curves were performed using the unbinned data from the counter.

DNA anchoring and sensing. Single stranded DNA (ssDNA) was anchored in the lipid bilayer by resuspending the dried DOPC lipids in a solution of $10 \mathrm{mM}$ tris(hydroxymethyl)aminomethane hydrochloride (Tris- $\mathrm{HCl}, \mathrm{pH}$ 8.0) containing the cholesterol modified oligonucleotides (Integrated DNA Technologies, Inc.). Unless specified, the molar ratio of lipid/DNA was 99.0:1.0. The cholesterol modification was placed at the 3' end of a 26 nucleotide oligonucleotide and featured a tetra(ethyleneglycol) (TEG) linker. The FRAP data was recorded using a cholesterol TEG linker on the 5' end of an 18-base oligonucleotide (5'-CholTEG/AGG CGC TGC CAG CGT CAT C-3'). The final lipid concentration of the solution was adjusted to $0.1 \mathrm{mg} / \mathrm{mL}$ with additional Tris- $\mathrm{HCl}$ keeping the $\mathrm{pH}$ at 8.0. Similar tip probe sonication procedures (as described above) were carried out on the DNA doped lipid vesicles to create unilamellar vesicles. Gel electrophoresis was used to verify there was no fragmentation of the DNA after sonication (see supporting information Figure S2). Identical procedures as described above were utilized to fuse the DNA doped lipid vesicles on the waveguides in the sealed and unsealed devices. We labeled the anchored single strands of DNA, after bilayer formation, for the confocal (unsealed devices) and annealing studies (sealed devices) with YOYO-1 (Invitrogen) and PicoGreen (Invitrogen), respectively.

For the real-time annealing experiments, Tris-buffered saline (TBS; $10 \mathrm{mM}$ Tris-HCl pH 8.0, $50 \mathrm{mM} \mathrm{NaCl}$ ) was used for hybridization and washing. After fusing the PicoGreen labeled vesicles, a liquid pulse train was pulled through the microchannel in the following order: lipid vesicles, TBS buffer, complementary target DNA (cDNA), TBS buffer. The cDNA solution had a DNA concentration of $15 \mu \mathrm{M}$ in TBS. Fluorescence generated by the 
evanescent field was monitored with a camera running at $\sim 2 \mathrm{~Hz}$ and capturing 200 sequential 512 x 512 pixel images. The sequences for the oligonucleotides were: 5'-TCC TGT GTC GAA TTG TTA TCC TTT TT/3cholTEG/-3'(probe DNA), and 5'-GGA TAA CAA TTC GAC ACA GGA-3' (target DNA)

\section{References.}

1. Pan, Z. W.; Dai, Z. R.; Wang, Z. L. Science 2001, 291, 1947-1949.

2. Sirbuly, D. J.; Law, M.; Pauzauskie, P.; Yan, H. Q.; Maslov, A. V.; Knutsen, K.; Ning, C. Z.; Saykally, R. J.; Yang, P. D. Proc. Natl. Acad. Sci. U.S.A 2005, 102, 7800-7805.

3. Snyder, A. W.; Love, J. D. Optical Waveguide Theory. Chapman and Hall: London, 1983. 
UCRL-JRNL-232761

Figure S1

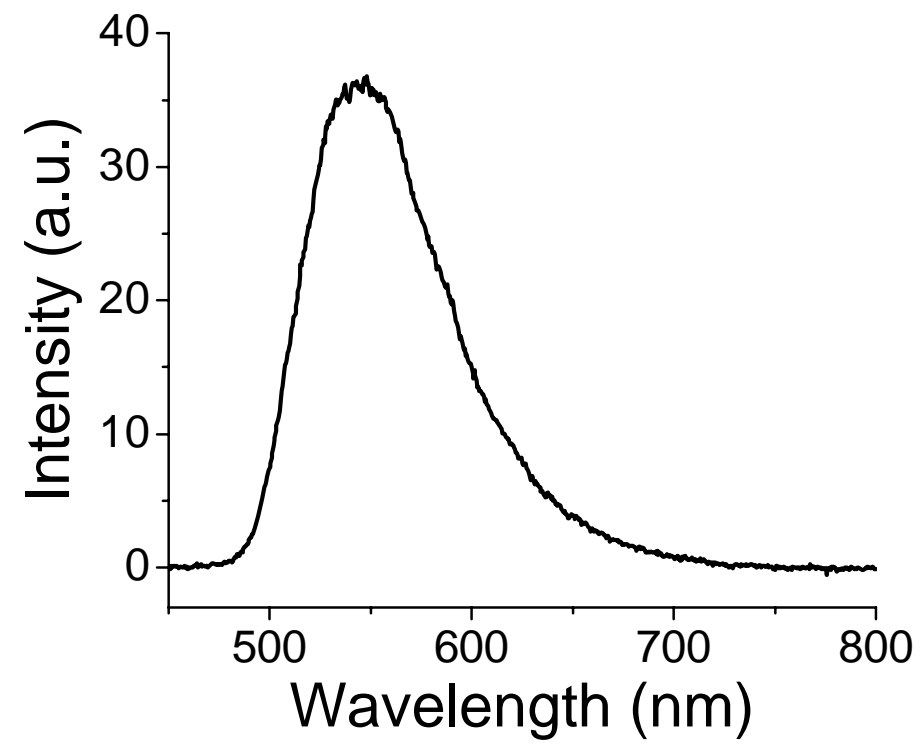

Figure S1. Spectrum capturing the NBDPE fluorescence generated by the evanescent field. 
UCRL-JRNL-232761

Figure S2

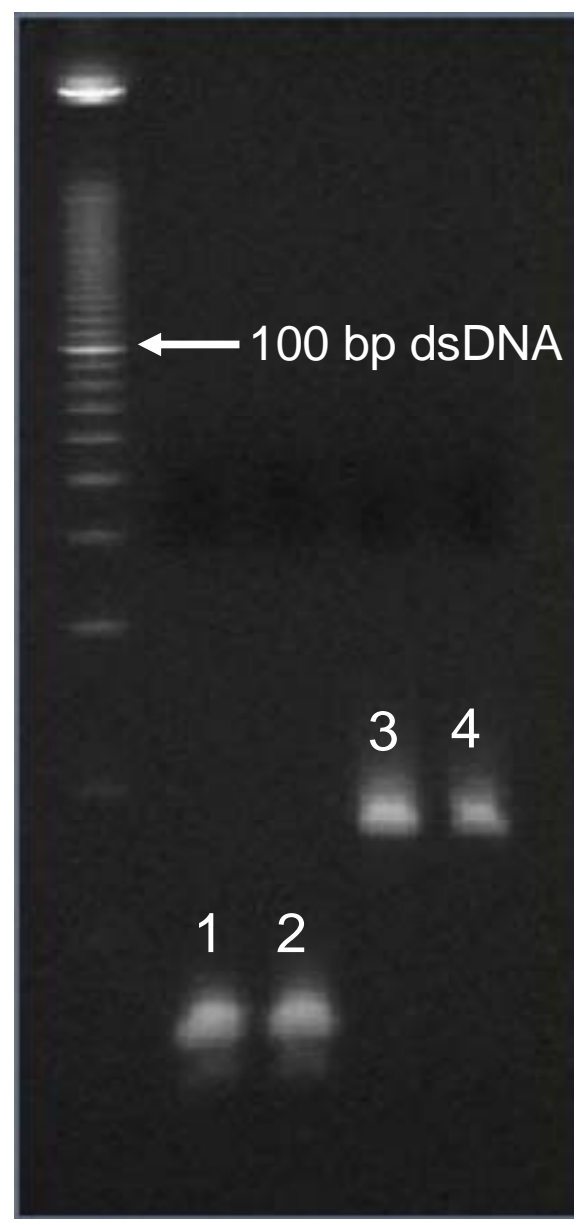

Figure S2. Gel electrophoresis of sonicated and unsonicated oligonucleotides. The unsonicated (1) and sonicated (2) probe ssDNA does not show any fragmentation after tip sonication. Similarly, the unsonicated (3) and sonicated (4) target cDNA does not show any signs of fragmenting during vesicle formation. 
Figure S3

A
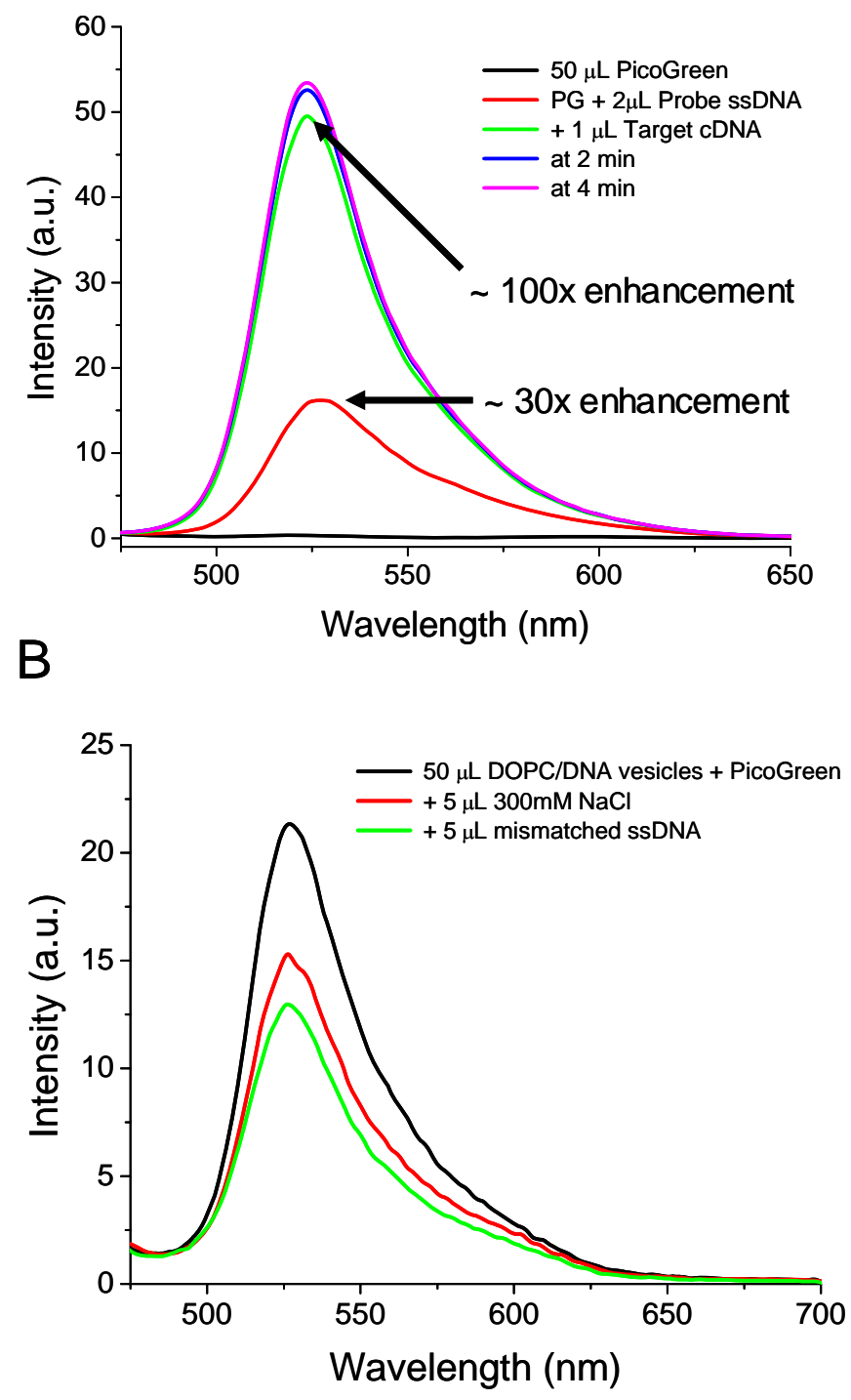

Figure S3. Fluorometry data of PicoGreen after incubating matched and mismatched target DNA with the probe ssDNA. (a) Fluorescence enhancement of PicoGreen upon addition of ssDNA and the complementary strand (salt was added to the PicoGreen solution). Only a slight increase in intensity is observed after additional 2 or 4 minute incubation periods. (b) Fluorescence data of PicoGreen labeled DOPC/DNA vesicles showing no enhancement, only dilution, after adding mismatched ssDNA. 